J. Austral. Math. Soc. 20 (Series A) (1975), 159-164.

\title{
GENERATORS OF REFLEXIVE ALGEBRAS
}

\author{
W. E. LONGSTAFF *
}

(Received 2 April 1973)

Communicated by J. B. Miller

\section{Introduction}

For any collection of closed subspaces of a complex Hilbert space the set of bounded operators that leave invariant all the members of the collection is a weakly-closed algebra. The class of such algebras is precisely the class of reflexive algebras as defined for example in Radjavi and Rosenthal (1969) and contains the class of von Neumann algebras. In this paper we consider the problem of when such algebras are finitely generated as weakly-closed algebras. It is to be hoped that analysis of this problem may shed some light on the famous unsolved problem of whether every von Neumann algebra on a separable Hilbert space is finitely generated. The case where the underlying space is separable and the collection of subspaces is totally ordered is dealt with in Longstaff (1974). In the present paper the result of Longstaff (1974) is generalized to the case of a direct product of countably many totally ordered collections each on a separable space. Also a method of obtaining non-finitely generated reflexive algebras is given.

The author wishes to thank Professor P. Rosenthal for his helpful suggestions concerning the content of this paper.

\section{Notation and preliminaries}

Throughout this paper the terms Hilbert space, subspace and projection will be used to mean complex Hilbert space, closed subspace and orthogonal projection respectively. The set of all bounded linear operators acting on a Hilbert space $H$ will be denoted by $B(H)$. The symbol ' $\subseteq$ ' will be used for set inclusion while ' $\subset$ ' will be reserved for proper inclusion. If $M$ and $N$ are subspaces and $M \subseteq N$ we denote by $N \ominus M$ the orthogonal complement of $M$ in $N$. The symbol ' $\oplus$ ' will always denote orthogonal direct sum. An operator $T \in B(H)$ is said to leave a subspace $M$ invariant if $T x \in M(x \in M)$. We indicate this by $T M \subseteq M$. If $P_{M}$

\footnotetext{
* The author is a Flinders University Research Fellow.
} 
denotes the projection with range $M$, the invariance of $M$ under $T$ is equivalent to $\left(I-P_{M}\right) T P_{M}=0$ where $I$ is the identity operator. Most of the notation, definitions and results we use concerning von Neumann algebras are to be found in Dixmier (1957). If $\left\{H_{\alpha}\right\}_{\alpha \in g}$ is a collection of Hilbert spaces, we denote by $\oplus_{\alpha \in g} H_{\alpha}$ their Hilbert space sum. If $H=\oplus_{\alpha} e g H_{\alpha}$ and $\left\{T_{\alpha}\right\}_{\alpha \in g}$ is a collection of operators satisfying $T_{\alpha} \in B\left(H_{\alpha}\right)(\alpha \in I)$ and $\sup _{\alpha \in I}\left\|T_{\alpha}\right\|<\infty$ the mapping $\left(x_{\alpha}\right)_{\alpha \in I} \rightarrow\left(T_{\alpha} x_{\alpha}\right)_{\alpha \in I}$ defines an element of $B(H)$, which we denote by $\oplus_{\alpha \in I} T_{\alpha}$. If $S_{\alpha}$ is a non-empty subset of $B\left(H_{\alpha}\right)(\alpha \in I)$ by $\oplus_{\alpha \in I} S_{\alpha}$ we mean the set of operators $\oplus_{\alpha \in I} T_{\alpha}$ with $T_{\alpha} \in S_{\alpha}(\alpha \in I)$.

If $\left\{N_{\alpha}\right\}$ is any collection of subspaces of the Hilbert space $H, \vee N_{\alpha}$ denotes the smallest subspace of $H$ containing each $N_{\alpha}$ and $\wedge N_{\alpha}$ denotes the largest subspace of $H$ contained in each $N_{\alpha}$. A collection $\mathscr{F}$ of subspaces of $H$ will be called complete if

(i) (0), $H \in \mathscr{F}$;

(ii) whenever $\phi \neq \mathscr{F}_{0} \subseteq \mathscr{F}, \wedge_{N \in \mathscr{F}_{0}} N \in \mathscr{F}$ and $\vee_{N \in \mathscr{F}_{0}} N \in \mathscr{F}$. If $\mathscr{S} \subseteq B(H)$ is any non-empty subset we let Lat $\mathscr{P}$ denote the set of all subspaces left invariant by every member of $\mathscr{S}$ and, for any non-empty collection $\mathscr{F}$ of subspaces of $H$ let $\operatorname{Alg} \mathscr{F}$ denote the set of all operators in $B(H)$ which leave every member of $\mathscr{F}$ invariant. It is not difficult to show that Lat $\mathscr{S}$ is a complete collection of subspaces, that $\mathrm{Alg} \mathscr{F}$ is a weakly-closed algebra of operators and that $\mathscr{F} \subseteq \operatorname{Lat} \operatorname{Alg} \mathscr{F}, \mathscr{S} \subseteq \mathrm{Alg} \operatorname{Lat} \mathscr{S}$. If $\mathscr{F}$ is any collection of subspaces of $H$, since the intersection of any family of complete collections is complete, it follows that there is a smallest complete collection containing $\mathscr{F}$, denoted by $\operatorname{co}(\mathscr{F})$. The following lemma generalizes Lemma 3.2 of Ringrose (1965).

LEMMA 2.1. If $\mathscr{F}$ is a non-empty collection of subspaces of a Hilbert space $H$ then

$$
\operatorname{Alg} \mathscr{F}=\operatorname{Alg} \operatorname{co}(\mathscr{F})=\operatorname{Alg} \text { Lat Alg } \mathscr{F} .
$$

Proof. Since $\mathscr{F} \subseteq$ Lat $\operatorname{Alg} \mathscr{F}$ and the latter is complete we have $\mathscr{F} \subseteq \operatorname{co}(\mathscr{F})$ $\subseteq$ Lat Alg $\mathscr{F}$ and so Alg Lat $\mathrm{Alg} \mathscr{F} \subseteq \operatorname{Alg} \operatorname{co}(\mathscr{F}) \subseteq \operatorname{Alg} \mathscr{F}$. The result follows from the fact that $\mathscr{S} \subseteq \mathrm{Alg}$ Lat $\mathscr{S}$ for any subset $\mathscr{S} \subseteq B(H)$.

The importance of this lemma is that, in the study of reflexive algebras i.e. algebras of the form $\operatorname{Alg} \mathscr{F}$, we may assume without loss of generality that $\mathscr{F}$ is a complete collection of subspaces.

If the collection $\mathscr{N}$ of subspaces is totally ordered by inclusion it is called a nest. Let $\mathscr{N}$ be a complete nest.

Definition 2.2. If $N \in \mathscr{N}$ define

$$
\begin{array}{ll}
N_{-}=\vee\{L \in \mathscr{N}: L \subset N\} & \text { if } N \neq(0) \\
N_{+}=\wedge\{L \in \mathscr{N}: N \subset L\} & \text { if } N \neq H
\end{array}
$$

and define $(0)_{-}=(0), H_{+}=H$. 
The definition of $N_{-}$is due to Ringrose (1965). With these definitions, it is obvious that $N_{-}, N_{+} \in \mathscr{N}$ and that $N_{-} \subseteq N \subseteq N_{+}$for every $N \in \mathscr{N}$. If $N_{-} \subset N$, $N_{-}$is called the immediate predecessor of $N$ in $\mathscr{N}$. If $N \subset N_{+}, N_{+}$is called the immediate successor of $N$ in $\mathscr{N}$. It is obvious that if $N \subset N_{+}$then $\left(N_{+}\right)_{-}=N$. If $M, N \in \mathscr{N}$ then the corresponding projections $P_{M}, P_{N}$ commute. Consequently, if $\mathscr{E}$ is the set of projections onto the members of $\mathscr{N}$ then the von Neumann algebra $\mathscr{C}$ generated by $\mathscr{E}$ is abelian. This von Neumann algebra is called the core of $\operatorname{Alg} \mathscr{N}$.

\section{A doubly generated reflexive algebra}

Let $\left\{H_{k}\right\}_{k=1}^{K}$ be a family of separable Hilbert spaces with $1 \leqq K \leqq \infty$. When $K=\infty$ in the following, the index set is to be taken as the set of positive integers. For every $k$ with $1 \leqq k \leqq K$ let $\mathcal{N}_{k}$ be a complete nest of subspaces of $H_{k}$. Let $H=\oplus_{k=1}^{K} H_{k}$ and let $\mathscr{F}$ be the collection of closed subspaces $N$ of $H$ of the form $N=\oplus{ }_{k=1}^{K} N_{k}$ where, for $1 \leqq k \leqq K, N_{k} \in \mathscr{N}_{k}$. Then Alg $\mathscr{F}$ is a weakly-closed sub-algebra of $B(H)$. We will prove the following.

THEOREM 3.1. AlgF is generated, as a weakly-closed algebra, by two operators.

Observe that $\operatorname{Alg} \mathscr{F}=\oplus_{k=1}^{K} \operatorname{Alg} \mathscr{N}_{k}$. Let $\mathscr{E}_{k}$ denote the collection of projections onto the members of $\mathscr{N}_{k}$ and $\mathscr{C}_{k}$ denote the core of Alg $\mathscr{N}_{k}$, for $1 \leqq k \leqq K$. As is shown in Longstaff (1974), for every $k$, it is possible to choose a maximal abelian self-adjoing algebra $\mathscr{D}_{k}$ and an operator $B_{k} \in \operatorname{Alg} \mathscr{N}_{k}$ such that

(i) $\mathscr{C}_{k} \subseteq \mathscr{D}_{k} \subseteq \operatorname{Alg} \mathscr{N}_{k}$;

(ii) whenever $(I-E) B_{k} E=0$ with $E$ a projection belonging to $\mathscr{D}_{k}$ then $E \in \mathscr{E}_{k}$.

Proof. Let $\mathscr{D}_{k}$ and $B_{k}$ be as above. Without loss of generality we may suppose that $\sup _{1 \leqq k \leqq K}\left\|B_{k}\right\|<\infty$. Let $B=\oplus_{k=1}^{K} B_{k} \in B(H)$ and $\mathscr{D}=\oplus_{k=1}^{K} \mathscr{D}_{k}$. Then $\mathscr{D}$ is a maximal abelian self-adjoint subalgebra of $B(H)$ (see Dixmier (1957; pages 12 and 19)) and $\mathscr{D} \subseteq \operatorname{Alg} \mathscr{F}, B \in \mathrm{Alg} \mathscr{F}$. Hence $\mathscr{F} \subseteq$ Lat $B \cap$ Lat $\mathscr{D}$. On the other hand, if $M$ is a subspace of $H$, which is invariant under $B$ and every element of $\mathscr{D}$ then $P_{M} \in \mathscr{D}$ and $\left(I-P_{+}\right) B P_{+}=0$. It follows that $P_{M}=\oplus_{k=1}^{K} E_{k}$ where $E_{k}$ is a projection belonging to $\mathscr{D}_{k}$ and that

$$
\left(I-E_{k}\right) B_{k} E_{k}=0 \quad(1 \leqq k \leqq K)
$$

Hence, $\quad E_{k} \in \mathscr{E}_{k}(1 \leqq k \leqq K)$ and $M=\oplus_{k=1}^{K} E_{k} H_{k} \in \mathscr{F}$. This shows that $\mathscr{F}=$ Lat $B \cap$ Lat $\mathscr{D}$.

Since $H$ is separable, by a well-known result of $\mathbf{J}$. von Neumann there is an operator $A \in \mathscr{D}$ such that $\mathscr{D}$ is the weakly-closed algebra generated by $A$. Let $\mathfrak{A}$ be the weakly-closed algebra generated by $A$ and $B$. Then $\mathfrak{A} \subseteq \mathrm{Alg} \mathscr{F}$ since $\mathrm{Alg} \mathscr{F}$ is weakly-closed, and so $\mathscr{F} \subseteq$ Lat $\mathfrak{A}$. But any subspace which is invariant under every element of $\mathfrak{A}$ is, in particular, invariant under $B$ and every element of $\mathscr{D}$ 
(since $\mathscr{D} \subseteq \mathfrak{A}$ ) and so belongs to $\mathscr{F}$. Thus Lat $\mathfrak{P}=\mathscr{F}$. Theorem 3 of Radjavi and Rosenthal (1969) then shows that $\mathfrak{A}=$ Alg $\mathscr{F}$. This completes the proof of the theorem.

\section{A non-finitely generated reflexive algebra}

Let $\mathscr{N}$ be a complete nest of subspaces of a Hilbert space $H$. If $\mathscr{F}=\{L: L$ a subspace of $H, N_{-} \subseteq L \subseteq N$ for some $\left.N \in \mathscr{N}\right\}$ then clearly $\mathscr{N} \subseteq \mathscr{F}$.

LEMMA 4.1. $\mathscr{F}=$ Lat Alg $\mathscr{F}$.

Proof. Obviously $\mathscr{F} \subseteq$ Lat Alg $\mathscr{F}$. Let $M \in$ Lat Alg $\mathscr{F}$ and let $N \in \mathscr{N}$ be arbitrary. We first show that either $M \subseteq N$ or $N \subseteq M$. Now $P_{N} \in \operatorname{Alg} \mathscr{F}$ for if $L \in \mathscr{F}$ and $K_{-} \subseteq L \subseteq K(K \in \mathscr{N})$ then either $K \subseteq N$ or $N \subset K$ from which we obtain, respectively, $L \subseteq N$ or $N \subseteq L$. In either case $P_{N} L \subseteq L$. Hence $P_{N} M \subseteq M$ and it follows that $P_{N} P_{M}=P_{M} P_{N}$ and that $M$ has the decomposition $M=(M \cap N)$ $\oplus(M \cap(H \ominus N))$. If $M \cap(H \ominus N)=(0)$ then $M \subseteq N$. If $M \cap(H \ominus N) \neq(0)$ we show that $N \subseteq M$. Let $e \in M \cap(H \ominus N)$ be a unit vector and let $f \in N$ be arbitrary. Then the operator $x \rightarrow(x \mid e) f(x \in H)$ which we denote by $e \otimes f$ belongs to $\operatorname{Alg} \mathscr{F}$ for if $L \in \mathscr{F}$, as we have seen either $L \subseteq N$ or $N \subseteq L$. In the first case, $(e \otimes f) L=(0) \subseteq L$ and in the second case, $(e \otimes f) L \subseteq N \subseteq L$. Since $M$ is invariant under every element of $A \lg \mathscr{F}$ we have $(e \otimes f) M \subseteq M$. But $e \in M$ and so $f=(e \otimes f)(e) \in M$. This shows that $N \subseteq M$. Thus $M$ is comparable with every element of $\mathscr{N}$. Let $N=\wedge\{L \in \mathscr{N}: M \subseteq L\}$. Then $N \in \mathscr{N}$ and $M \subseteq N$. If $N=(0)$ then $M=(0) \in \mathscr{F}$. If $N \neq(0)$ and $L \in \mathscr{N}$ with $L \subset N$ then $M \subseteq L$ cannot be true and so $L \subset M$. Thus $N_{-}=\bigvee\{L \in \mathscr{N}: L \subset N\} \subseteq M$ and $M \in \mathscr{F}$. This completes the proof of the lemma.

THEOREM 4.2. If $N$ is an element of $\mathscr{N}$ with $N_{-} \subset N \subset N_{+}$then $\mathrm{Alg} \mathscr{F}$ cannot be generated as a weakly-closed algebra by fewer than $\operatorname{dim}\left(N \ominus N_{-}\right)-1$ elements.

Proof. Let $k=\operatorname{dim}\left(N \ominus N_{-}\right)$. Then $k \geqq 1$. We may suppose that $k>2$. Let $A_{1}, A_{2}, \cdots, A_{n}$ be any finite set of elements of Alg $\mathscr{F}$ with $n<k-1$ and let $\mathfrak{A}$ be the weakly-closed algebra they generate. Then $\mathfrak{A} \subseteq A \lg \mathscr{F}$ since Alg $\mathscr{F}$ is weaklyclosed, and so $\mathscr{F} \subseteq$ Lat $\mathfrak{A}$. To show that $\mathfrak{U} \subset A \lg \mathscr{F}$ it suffices by the previous lemma to show that $\mathscr{F} \subset$ Lat $\mathfrak{A}$. Let $x \in N_{+} \backslash N$ be arbitrary and let $[x]$ be the one-dimensional subspace spanned by $x$. Now $N \subseteq N \vee[x] \subseteq N_{+}$and $\left(N_{+}\right)_{-}$ $=N$. Thus $N \vee[x] \in \mathscr{F}$ and so is invariant under $A_{i}$ for $i=1,2, \cdots, n$. It follows that for every $i$, there exist $\alpha_{i} \in \mathbb{C}$ and $m_{i} \in N$ such that $A_{i} x=\alpha_{i} x+m_{i}$. Let $M=N_{-} \vee\left\{x, m_{1}, m_{2}, \cdots, m_{n}\right\}$. Then since $N_{-} \subseteq N_{-} \vee\left\{m_{1}, m_{2}, \cdots, m_{n}\right\} \subseteq N$, it follows that $A_{j}\left(N_{-} \vee\left\{m_{1}, m_{2}, \cdots, m_{n}\right\}\right) \subseteq N_{-} \vee\left\{m_{1}, m_{2}, \cdots, m_{n}\right\}$ for $1 \leqq j \leqq n$. Thus $M$ is invariant under every $A_{j}$ and hence $M \in$ Lat $\mathcal{A}$. But $M \notin \mathscr{F}$ for if $L_{-} \subseteq M \subseteq L$ for some $L \in \mathscr{N}$ then either $L \subseteq N$ or $N \subset L$. The former cannot be true since $x \notin N$. Hence $N \subseteq L$ and $N \subseteq L_{-} \subseteq M$. But $N \subseteq M$ cannot be true 
either for if so then $N \ominus N_{-} \subseteq M \ominus N_{-}$and so $\operatorname{dim}\left(N \ominus N_{-}\right)=k \leqq$ $\operatorname{dim}\left(M \ominus N_{-}\right) \leqq n+1$ and this contradicts the fact that $n<k-1$. Thus $\mathfrak{A} \subset \operatorname{Alg} \mathscr{F}$ and the proof of the theorem is complete.

Corollary 4.3. If $\mathscr{G}=\left\{N \in \mathscr{N}: N_{-} \subset N \subset N_{+}\right\}$and $\sup _{N=\mathscr{G}} \operatorname{dim}\left(N \ominus N_{-}\right)$ $=\infty$ then $\mathrm{Alg} \mathscr{F}$ is not finitely generated as a weakly-closed algebra.

Corollary 4.4. If for some $N \in \mathscr{N}, N_{-} \subset N \subset N_{+}$and $\operatorname{dim}\left(N \ominus N_{-}\right)=\infty$ then $\mathrm{Alg} \mathscr{F}$ is not finitely generated as a weakly-closed algebra.

A particular instance of this latter corollary, the case where $\mathscr{N}$ is the nest with three elements (0), $M, H$ where (0) $\subset M \subset H$ and $\operatorname{dim} M=\infty$, has already been established in Longstaff (1974).

\section{Some additional results}

In the previous section, starting with a complete nest $\mathscr{N}$ we saw that $\mathscr{F}=\left\{L: N_{-} \subseteq L \subseteq N\right.$ for some $\left.N \in \mathscr{N}\right\}$ was a complete collection of subspaces such that $\mathscr{N} \subseteq \mathscr{F}$ and such that every element of $\mathscr{F}$ is comparable with every element of $\mathscr{N}$. In a sense we can reverse the procedure. More precisely we prove

Proposition 5.1. I. If $\mathscr{N}$ is a complete nest and

$$
\mathscr{F}=\left\{M: M \text { a subspace, } N_{-} \subseteq M \subseteq N \text { for some } N \in \mathscr{N}\right\}
$$

then $\mathscr{F}$ is a complete collection of subspaces containing $\mathscr{N}$ and

$$
\mathscr{N}=\{N \in \mathscr{F}: N \text { is comparable with every element of } \mathscr{F}\} .
$$

II. If $\mathscr{F}$ is a complete collection of subspaces then

$$
\mathscr{N}=\{N \in \mathscr{F}: N \text { is comparable with every element of } \mathscr{F}\}
$$

is a complete nest contained in $\mathscr{F}$ and

$$
\mathscr{F} \subseteq\left\{M: M \text { a subspace, } N_{-} \subseteq M \subseteq N \text { for some } N \in \mathscr{N}\right\} .
$$

Proof. I. $\mathscr{F}$ is complete for by Lemma $4.1, \mathscr{F}=\operatorname{co}(\mathscr{F})=$ Lat Alg.F्F. It o.nly remains to show that if $N \in \mathscr{F}$ and $N$ is comparable with every element of $\mathscr{F}$ then $N \in \mathscr{N}$. Since $N \in \mathscr{F}, L_{-} \subseteq N \subseteq L$ for some $L \in \mathscr{N}$. If $N \notin \mathscr{N}$ then $L_{-} \subset N \subset L$. Now $L_{-} \subseteq L_{-} \oplus(L \ominus N) \subseteq L$ and so $L_{-} \oplus(L \ominus N) \in \mathscr{F}$. But $N$ and $L_{-} \oplus(L \ominus N)$ are not comparable. This contradiction shows that $N \in \mathscr{N}$.

II. Obviously $\mathscr{N} \subseteq \mathscr{F}$ and $\mathscr{N}$ is a nest containing $(0)$ and $H$. Let $\mathscr{N}_{0}$ be any non-empty subset of $\mathscr{N}$ and let $M=\bigvee_{N \in \mathscr{N}_{0}} N$. Then $M \in \mathscr{F}$ since $\mathscr{F}$ is complete. If $L \in \mathscr{F}$ then every element of $\mathscr{N}_{0}$ is comparable with $L$. If $N \subseteq L$ for every $N \in \mathscr{N}_{0}$ then $M \subseteq L$. If this is not the case then $L \subset N$ for some $N \in \mathscr{N}_{0}$ and so $L \subset M$. Hence $M \in \mathscr{N}$. A similar argument applies to $\wedge_{N \in \mathscr{N}_{0}} N$. Thus $\mathscr{N}$ is a complete nest. Now let $M \in \mathscr{F}$ and let $N=\wedge\{L \in \mathscr{N}: M \subseteq L\}$. Then $N \in \mathscr{N}$ and 
$M \subseteq N$. If $N=(0)$ then certainly $N_{-} \subseteq M \subseteq N$. If $L \in \mathscr{N}$ and $L \subset N$ then $M \subseteq L$ is impossible so $L \subset M$ and $N_{-}=\vee\{L \in \mathscr{N}: L \subset N\} \subseteq M$. Thus $N_{-} \subseteq M \subseteq N$. This completes the proof of the lemma.

\section{References}

J. Dixmier (1957), Les algèbres d'opérateurs dans l'espace Hilbertien (Gauthier-Villars, Paris, 1957.)

W. E. Longstaff (1974), 'Generators of nest algebras', Canad. J. Math. 26, 565-575.

H. Radjavi and P. Rosenthal (1969), 'On invariant subspaces and reflexive algebras', Amer. J. Math. 91, 683-692.

J. R. Ringrose (1965), 'On some algebras of operators', Proc. London Math. Soc. (3) 15, 61-83.

The Flinders University of South Australia

Bedford Park, 5042

South Australia 